\title{
Possible role of autoantibodies in the pathophysiology of GM2 gangliosidoses
}

\author{
Akira Yamaguchi, ${ }^{1}$ Kayoko Katsuyama, ${ }^{1}$ Kiyotaka Nagahama, ${ }^{1}$ Toshiyuki Takai, ${ }^{2}$ \\ Ichiro Aoki, ${ }^{1}$ and Shoji Yamanaka ${ }^{1}$ \\ ${ }^{1}$ Department of Pathology, Yokohama City University School of Medicine, Yokohama, Japan \\ ${ }^{2}$ Department of Experimental Immunology and Core Research for Evolution Science and Technology Programme of Japan \\ Science and Technology Corp., Institute of Development, Aging and Cancer, Tohoku University School, Sendai, Japan
}

\begin{abstract}
Mice containing a disruption of the Hexb gene have provided a useful model system for the study of the human lysosomal storage disorder known as Sandhoff disease (SD). Hexb $b^{-/}$mice rapidly develop a progressive neurologic disease of ganglioside GM2 and GA2 storage. Our study revealed that the disease states in this model are associated with the appearance of antiganglioside autoantibodies. Both elevation of serum antiganglioside autoantibodies and IgG deposition to CNS neurons were found in the advanced stages of the disease in $\mathrm{Hexb}^{-/-}$mice; serum transfer from these mice showed IgG binding to neurons. To determine the role of these autoantibodies, the Fc receptor $\gamma$ gene $(F c R \gamma)$ was additionally disrupted in $\mathrm{Hexb}^{-/-}$mice, as it plays a key role in immune complex-mediated autoimmune diseases. Clinical symptoms were improved and life spans were extended in the $\mathrm{Hexb}^{-/-} \mathrm{FcR} \gamma^{/-}$mice; the number of apoptotic cells was also decreased. The level of ganglioside accumulation, however, did not change. IgG deposition was also confirmed in the brain of an autopsied SD patient. Taken together, these findings suggest that the production of autoantibodies plays an important role in the pathogenesis of neuropathy in SD and therefore provides a target for novel therapies.
\end{abstract}

J. Clin. Invest. 113:200-208 (2004). doi:10.1172/JCI200419639.

\begin{abstract}
Introduction
Lysosomal storage disorders (LSDs) arise from functional defects in one or more of the proteins essential to normal lysosome function. This typically involves the enzymes that play a critical role in the intracellular digestion of glycoproteins, glycolipids, glycosaminoglycans, or other macromolecules (1). GM2 gangliosidoses, one of the major LSDs, are caused by an abnormality in the hexosaminidases (Hex's) $(1,2)$. Hex A consists of a heterodimer of a $\beta$-subunit (HEXA gene product) and an $\alpha$-subunit (HEXB gene product). Hex $B$ is a homodimer of $\beta$-subunits. Mutations in the HEXA gene cause Tay-Sachs disease, whereas mutations in the HEXB gene cause Sandhoff disease (SD) (1).

Mice with disruptions in the Hexb gene develop an SD-like illness and therefore have provided a useful model for investigating the pathophysiology of SD
\end{abstract}

Received for publication July 29, 2003, and accepted in revised form November 11, 2003.

Address correspondence to: Shoji Yamanaka, Department of Pathology, Yokohama City University School of Medicine, 3-9 Fukuura, Kanazawa-ku, Yokohama, Kanagawa 236-0004, Japan. Phone: 81-45-787-2586; Fax: 81-45-786-0191;

E-mail: shojiy@med.yokohama-cu.ac.jp.

Conflict of interest: The authors have declared that no conflict of interest exists.

Nonstandard abbreviations used: lysosomal storage disorder (LSD); hexosaminidase (Hex); Sandhoff disease (SD); bone marrow transplantation (BMT); $\mathrm{Fc}$ receptor $(\mathrm{FcR})$; highperformance, thin-layer chromatography (HPTLC); propidium iodine (PI); blood-brain barrier (BBB); systemic lupus erythematosus (SLE); death-associated protein 6 (DAXX).
(3-5). Neurologic dysfunction is the major clinical manifestation of GM2 gangliosidoses, correlating closely with the severity of the illness. These neurological abnormalities have been ascribed in part to neuronal cell death caused by the accumulation of both undigested GM2 gangliosides and related lipids in neuronal lysosomes (6). However, several recent investigations have suggested that ganglioside accumulation in neurons alone cannot completely explain the nerve cell damage and the short life span that characterizes SD $\left(\mathrm{Hexb}^{-/-}\right)$mice. It was previously reported that bone marrow transplantation (BMT) from normal $\left(\mathrm{Hexb}^{+/+}\right)$to SD mice suppresses neuronal death and improves survival ratios despite having no effect on either Hex activity or ganglioside accumulation in the brain $(7,8)$. Certain inflammatory processes, including microglial activation, indicated by cytokine expression were also observed (8-10). In addition, other recent studies indicated that antiganglioside antibodies were present in several neuropathies, including GuillainBarré syndrome, amyotrophic lateral sclerosis, and Fisher syndrome (11-13).

Thus, we hypothesized that substrates such as GM2 and/or GA2 could evoke autoimmune responses because they could not easily be degraded or cleared due to specific enzyme deficiencies. As a consequence of this, autoantibodies would bind to antigens on the cell surface of neurons and trigger microglial activation via the $\mathrm{Fc}$ receptor $(\mathrm{FcR})$ common $\gamma$-chain. Studies were undertaken to evaluate this hypothesis. 


\section{Methods}

Mice. All mice used in this study were bred and housed under standard nonsterile conditions. All animal studies were approved by the Animal Committee at Yokohama City University. SD mice (Hexb-/mice; C57BL/6X129/Sv background) were kindly provided by R. L. Proia (NIH) and were bred in a closed colony over 30 generations so that they were inbred for C57BL/6- and 129/Sv-derived genes (4). FcR $\gamma$ gene knockout mice (FcR $\gamma^{/-}$mice) (14) with C57BL/ 6 were bred with Hexb $b^{-/}$mice to obtain doubly heterozygous $\left(\mathrm{Hex}^{+/-} \mathrm{FcR} \gamma^{+/-}\right)$mice. To minimize variations due to differences in genetic background, all genotypes used in this study were derived from these cross-breedings, and the sib pairs were used as a comparison. The bexb and $F c R \gamma$ genotypes were determined by PCR using tail DNA (15). Primer sequences used for $F c R \gamma$ were as follows: (1) $5^{\prime}$-GCCCTGGGAGAGCCGCAGCTCTGCTATATCCTGGA-3', (2) $5^{\prime}$-CTCACGGCTGGCTATAGCTGCCTTTCGGACCTGGA$3^{\prime}$. In the $F c R \gamma^{\prime-}$ and WT genotypes, $1.2-\mathrm{kb}$ and $0.24-$ $\mathrm{kb}$ bands were observed, respectively. After denaturation at $94^{\circ} \mathrm{C}$ for 1 minute, the $\mathrm{PCR}$ reaction was cycled 30 times at $94^{\circ} \mathrm{C}$ for 30 seconds and $68^{\circ} \mathrm{C}$ for 5 minutes. PCR products were separated by electrophoresis through a $2 \%(\mathrm{w} / \mathrm{v})$ agarose gel and visualized by ethidium bromide staining.

Antibodies. A rabbit IgG antibody against mouse GA2 was obtained from Dia-iatron (Tokyo, Japan). Biotin-conjugated anti- $N$-acetyl GM2 antibody that recognizes mouse GM2 was obtained from Seikagaku Corp. (Tokyo, Japan). Alkaline phosphatase-labeled goat antimouse IgG antibody, which also recognizes human IgG, was obtained from Southern Biotechnology Associates Inc. (Birmingham, Alabama, USA). Alexa fluor goat antirabbit IgG and fluor donkey antigoat IgG were obtained from Molecular Probes (Eugene, Oregon, USA). Streptavidin-FITC-conjugated GM2 was purchased from GIBCO BRL (Tokyo, Japan). Horseradish peroxidaseconjugated goat antimouse IgG was obtained from ICN Pharmaceuticals Inc. (Costa Mesa, California, USA).

Human brain tissue. A human brain sample, taken from a 2-year-old boy with SD who was autopsied at Nagoya City University Medical School, was kindly provided by $M$. Tatematsu (16). This experiment was approved by the Ethical Committee of Yokohama City University School of Medicine.

Extraction and analysis of glycolipids. Glycolipids were extracted from mouse brain as described previously (4), applied to high-performance, thin-layer chromatography (HPTLC) plates (Silicagel 60, Merck, Darmstadt, Germany), and chromatographed with chloroform/ methanol/0.22\% aqueous $\mathrm{CaCl} 2$ (60:40:9, v/v/v). The glycolipids were then visualized by spraying the HPTLC plates with anthrone-sulfuric acid reagent followed by heating on a hot plate.

ELISA. Antiganglioside antibodies were determined by ELISA assays as described previously (17). Briefly, flat-bottom plates (Greiner Immunoplate, Fricken- hausen, Germany) were coated with appropriately diluted soluble antigens (extracted from Hexb ${ }^{-1-}$ mouse brain and liver) and then blocked with $1 \%$ BSA in PBS. Serum diluted in PBS containing 1\% BSA was incubated on antigen-coupled plates for 2 hours. Unbound immunoglobulin was washed away with PBS containing $0.05 \%$ Tween 20 . Horseradish peroxidase-conjugated goat antimouse IgG was added for a further 2 hours and the plates were washed again. A color reaction was obtained by adding orthophenylenediamine$\mathrm{HCl}$ substrates (Wako, Tokyo, Japan). The data were determined by OD values at $405 \mathrm{~nm}$ with reference to the OD of normal WT mouse (C57BL/129) serum. Serum cytokines were detected by the ELISA method using a Mouse Interleukin-4 ELISA Kit (Endogen Inc., Rockford, Illinois, USA) and a Mouse Interferon Gamma ELISA Kit (Endogen Inc.).

Immunofluorescence study. Organs were removed from the necropsied animals and processed for frozen or paraffin sections. The sections were pretreated with PBS containing 3\% BSA (Nissui, Tokyo, Japan) for 15 minutes and incubated overnight with antibody at $4^{\circ} \mathrm{C}$. For double staining, the sections were incubated with propidium iodine (PI) solution for nuclear staining after the secondary fluorescent antibody reaction. Stained sections were observed using laser scanning microscopy (LSM 101, Olympus, Tokyo, Japan).

Immunohistochemistry. For the detection of IgG deposition, alkaline phosphate-labeled goat antimouse IgG antibody was used for both mouse and human brain tissue samples using a Histofine kit according to the manufacturer's instructions (Nichirei Corp., Tokyo, Japan).

Serum transfer experiment with blood-brain barrier disruption. D-Mannitol was chosen as a hypertonic agent to open the blood-brain barrier (BBB) osmotically. The safe limits for its experimental use in animals has been described elsewhere $(18,19)$. Mannitol was dissolved in PBS at a concentration of $1.6 \mathrm{~mol} / 1$ and kept at $37^{\circ} \mathrm{C}$. Three hundred microliters of pooled sera from either WT $\left(\mathrm{Hexb}^{+/+}\right)$or $\mathrm{Hexb}^{-/-}$mice that were between 14 and 15 weeks of age was injected into the tail vein of 8 -week-old SCID mice with 200 $\mu \mathrm{l}$ of $1.6 \mathrm{~mol} / \mathrm{l} \mathrm{D}$-mannitol. The mice were killed 30 minutes after the injections.

Apoptosis analysis. Apoptotic cells were detected by the in situ TUNEL method using a DeadEnd Colorimetric TUNEL system (Promega, Madison, Wisconsin, USA). An apoptotic DNA ladder was detected using a TACS Apoptotic DNA Laddering Kit and Tissue Supplement Reagents (Trevigen Inc., Gaithersburg, Maryland, USA). One microgram of extracted DNA taken from the brain of a 14-week-old mouse was subjected to electrophoresis on a $2 \%(\mathrm{w} / \mathrm{v})$ agarose gel containing ethidium bromide $(0.4 \mathrm{mg} / \mathrm{ml})$.

Behavioral testing. Motor function and balance were measured with a standard rotorod apparatus (Ugo Basile 7650; BM Apparatus, Tokyo, Japan). The machine was set to an initial speed of $4 \mathrm{rpm}$, and the acceleration was increased by $2 \mathrm{rpm} / \mathrm{min}$. The latency 

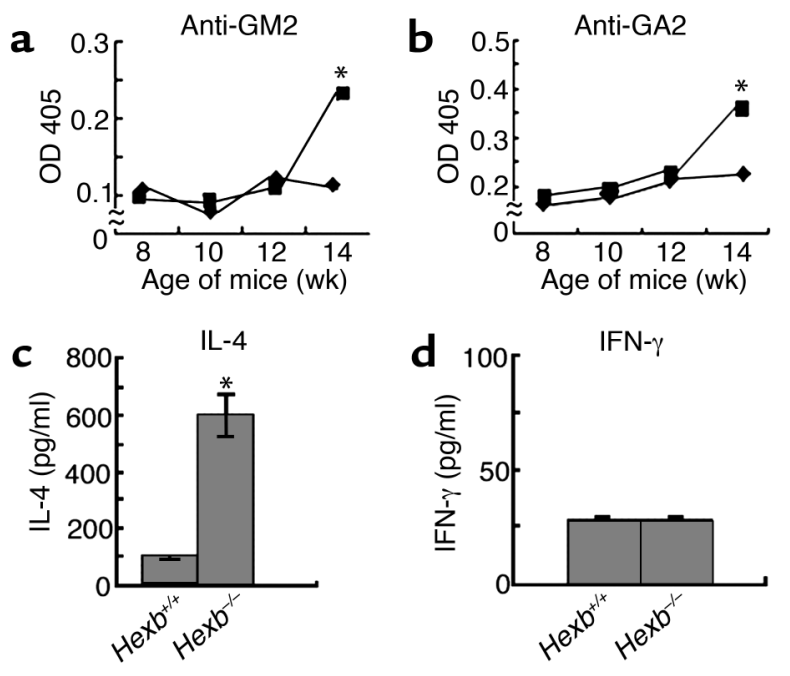

to fall off from the rotating cylinder was also measured. Mice that fell within 15 seconds were given a second trial, and those that did not fall during a 250-second period were given a score of 250 seconds. Rotorod

\section{Figure 1}

Detection of anti-GM2 and-GA2 antibodies, IL- 4 and INF- $\gamma$ by ELISA. Autoantibodies against GM2 (a) and GA2 (b) in serum were measured in $\mathrm{Hexb}^{-/-}$(squares) and Hexb ${ }^{+/+}$(diamonds) mice. Serum IL-4 (c) and INF- $\gamma($ d) levels were measured in 14-week-old Hexb-/- and Hex $b^{+/+}$mice. $n=$ eight mice per group. ${ }^{*} P<0.01$, Student's $t$ test.

testing was conducted once a week for 8-week-old mice until the time when they could no longer walk due to gait apraxia.

Clinical scores. Neurological activity in mice was scored using the following criteria: a score was assigned for the effect when a mouse was lifted by the tail and also for the ability to roll over when the mouse was left in a dorsal position. Each score was graded into the following five categories: 0, normal: limbs extended and body twisted, able to roll back to prone from supine position; 1 , slight: limbs extended with slight tremor and twisted body, able to roll back to prone from supine position; 2 , mild: limbs extended without twisted body, able to roll back to prone $\mathbf{a}$
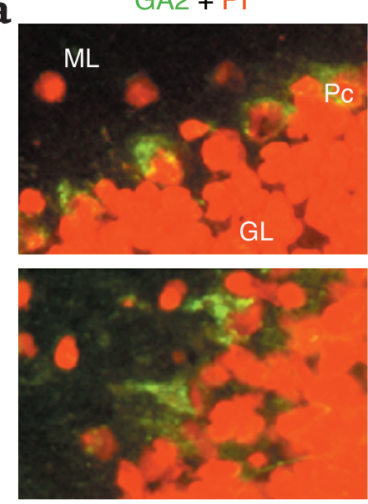

C

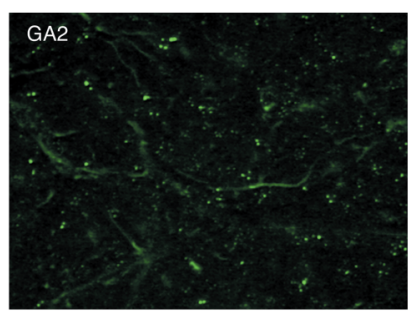

$\lg G+P \mid$
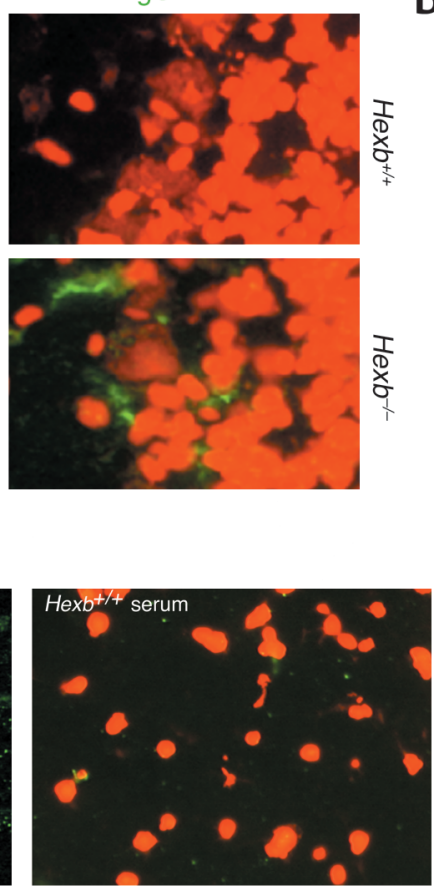

b
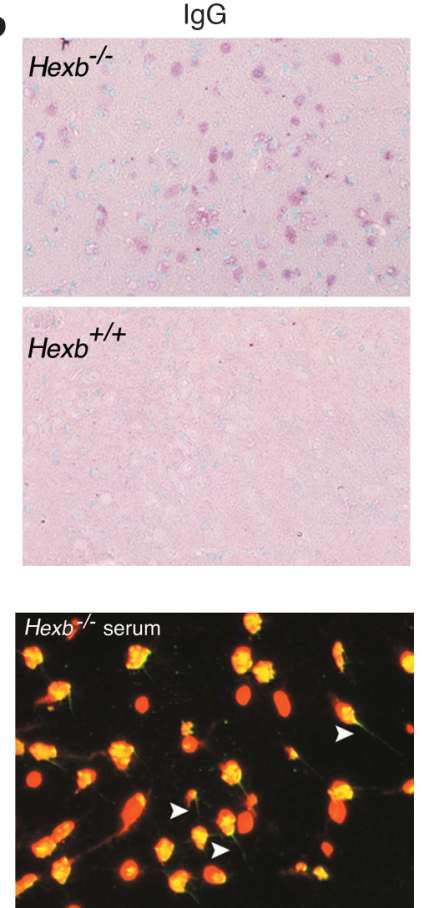

\section{Figure 2}

Deposition of IgG in the brain. (a) Immunofluorescent staining with anti-GA2 antibody (green) and nuclear staining with PI (red) is shown for frozen brain sections. Both cell surface expression and intracellular storage of GA2 in the cerebella of 14-week-old Hex $b^{+/+}$mice and $\mathrm{Hexb}^{-/-}$mice are shown (left panel). ML, molecular layer; Pc, Purkinje cells; GL, granular layer. The presence of IgG detected by anti-lgG antibody (green) is shown in the same area (right panel). Representative sections with three mice per group are shown. IgG is detected only in Hexb-/- mice. Original magnification, $\times 400$. (b) Immunohistochemical staining with alkaline phosphatase-labeled anti-IgG antibody shows $\operatorname{lgG}$ deposition in the neurons of the thalamus (red) of 14-week-old Hexb-/- mice with no IgG deposition evident in the thalamus of agematched $\mathrm{Hexb}^{+/+}$mice. Representative sections are shown. Methylgreen was used for counterstaining. Original magnification, $\times 200$. (c) Deposition of IgG present in the hippocampal commissure of BBB-disrupted SCID mice following injection of 14-week-old Hexb-/- mice serum. Prior to injection, the presence of the GA2 (stained green) was confirmed in SCID mice (left). Deposition of IgG was investigated following BBB disruption. Serum transfer from the $\mathrm{Hexb}^{+/+}$mice did not reveal IgG deposition (middle), whereas the presence of IgG was demonstrated in the same area of the SCID mice injected with Hexb-/- mice sera (right). Green, presence of Alexa-labeled GA2 (left) or Alexa-labeled IgG (middle and right); red, nuclear staining with PI. Arrows indicate axons or dendrites that were stained green. Original magnification, $\times 400$. 
from supine position; 3 , moderate: flexed and paws clenched, able to roll back to prone from supine position; 4, severe: flexed and paws clenched, unable to roll back to prone from supine position.

Data analysis. A Student's $t$ test was employed to evaluate the significance of the data. $P<0.05$ was considered statistically significant.

\section{Results}

Serum anti-GM2 and GA2 antibodies are elevated in aged Hexb $b^{-/}$mice. First, we conducted experiments according to our proposed hypothesis that autoantibodies are associated with the neuronal damage and symptoms of gangliosidoses. Autoantibodies for the gangliosides GM2 and GA2 were therefore evaluated by serum ELISA. Significant elevation of serum antibody levels was observed in the terminal stages of $\mathrm{Hexb}^{-/-}$diseased mice that were more than 14 weeks old (Figure 1, a and b), which was consistent with our hypothesis. Associated with this autoantibody production, the level of serum IL-4 (Figure 1c), but not IFN- $\gamma$ (Figure 1d), was also elevated, suggesting that certain immune and inflammatory processes may be occurring in aged $\mathrm{Hex}^{-/-}$mice. Thus, the release of autoantigens from ganglioside-accumulating cells may be involved in the production of autoantibodies.

IgG deposition is observed on the cell surface of neurons in the CNS of Hexb ${ }^{-1-}$ mice, but not WT mice. Next we evaluated the presence of autoantibodies in the CNS. Tissue sections from various CNS sites were taken from 14-weekold $\mathrm{Hexb}^{-/-}$mice and analyzed for GA2 accumulation and IgG deposition (Figure 2a). Immunohistochemistry revealed that the Purkinje cells of both WT and Hex $b^{-/-}$mice showed specific expression of GA2 on their cell surfaces. In contrast to Purkinje cells in WT mice, however, those in the Hexb $b^{-/}$mice simultaneously showed IgG deposition. Similar findings were observed in various sites of the CNS including the thalamus, where extensive apoptosis is known to occur (Figure $2 \mathrm{~b}$ ), as has been reported previously (8).

Serum transfer from $\mathrm{Hexb}^{-1-}$ mice to BBB-disrupted mice reveals the presence of antibodies that bind to neurons. To confirm the presence of autoantibodies that can bind to the cell surface of neurons, we disrupted the BBB of SCID mice by D-mannitol and injected sera from either $\mathrm{Hexb}^{-/-}$or WT mice. SCID mice were used in this experiment, since they have low background levels of immunoglobulins. Expression of GA2 was confirmed in the hippocampal commissure of SCID mice (Figure $2 c)$. Following the disruption of BBB and the transfer of serum from either WT or Hexb $b^{-/-}$mice, the hippocampal commissure areas of the SCID mice were analyzed for immunoglobulin binding using anti-IgG antibodies. IgG-bearing neurons were seen in the SCID mice that had been injected with $H e x b^{-1-}$ serum, whereas SCID mice injected with WT serum did not show IgG binding either on neuronal cell surfaces or in the cytoplasm (Figure $2 \mathrm{c}$ ). It is noteworthy that a previous study reported altered BBB permeability in $\mathrm{Hexb}^{-/-}$ mice (10). Our results indicate that autoantibodies move to the brain parenchyma via $\mathrm{BBB}$ and bind to the cell surface of neurons in $\mathrm{Hexb}^{-/-}$mice.

Double-knockout $\left(\mathrm{Hexb}^{-/-} \mathrm{FcR} \mathrm{\gamma}^{-/-}\right)$mice show better clinical outcomes and lower GA2 antibody levels than Hexb-/$F c R \gamma^{\dagger /+}$ mice. It was difficult to determine the relationship between $\operatorname{IgG}$ deposition and apoptosis, since apoptosis was limited to specific areas whereas IgG deposition was seen diffusely. To determine the role of autoantibodies more precisely, we generated mice lacking both the FcR $\gamma$ gene and the Hexb gene by intercrossing (14). The double-knockout genotypes were confirmed by PCR of tail DNA (Figure 3a). As seen in Figure $3 \mathrm{~b}$, the $\mathrm{Hexb}^{-/-F c R \gamma^{1 /+}}$ mouse at 14 weeks old was less active, quite lean, and displayed muscle atrophy with wide-opening hind limbs to support the body, whereas the $\mathrm{Hexb}^{-1-} \mathrm{FcR} \gamma^{\prime-}$ mouse of the same age was active and mobile. Serum antibodies against GA2 were also evaluated in the $H e x b^{-/-F c R \gamma^{/-}}$mice. A significant decrease in serum antibody levels over $H e x b^{-/-} F c R \gamma^{1 /+}$ mice was noted in 14-week-old $H e x b^{-/-} F c R \gamma^{/-}$mice,
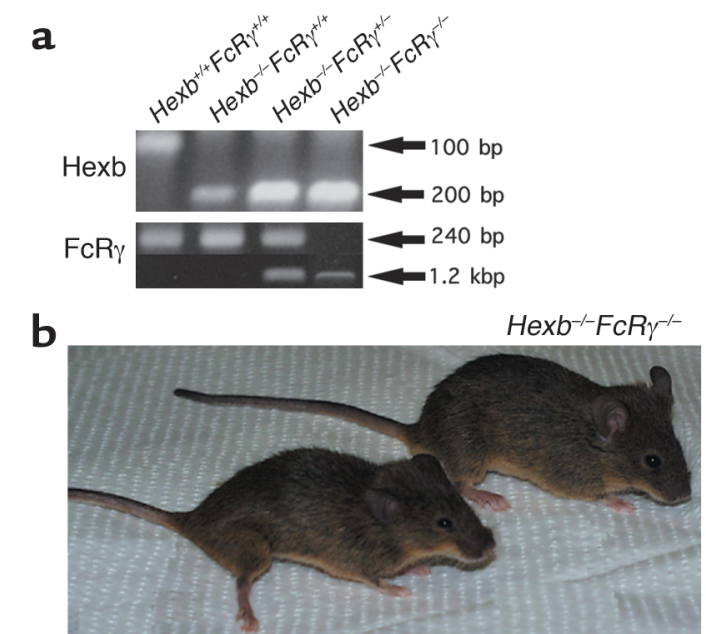

$\mathrm{Hexb}^{-1-} \mathrm{FcR} \gamma^{+/+}$

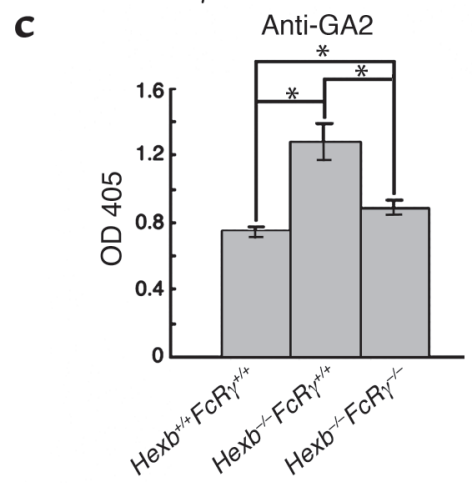

Figure 3

Generation of $\mathrm{Hexb}^{-/-} \mathrm{FcR \gamma ^{-/ }}$ mice. (a) PCR analysis of mouse tail DNA. From left lane: $\mathrm{Hexb}^{+/+} F c R \gamma^{+/+}, \mathrm{Hexb}^{-/-} \mathrm{FcR} \gamma^{/+}, \mathrm{Hexb^{-/- }} F \mathrm{CR} \gamma^{+/-}$, and $H e x b^{-/-} F_{c} R \gamma^{-1-}$ mice. (b) Clinical appearance of Hexb $b^{-/-} F c R \gamma^{+/+}$ and $\mathrm{Hexb}^{-/-} \mathrm{F}_{\mathrm{CR}} \gamma^{-/}$mice at 14 weeks. (c) Investigation of the titers of anti-GA2 antibodies in 14-week-old mice. Anti-GA2 antibody was at lower levels in $\mathrm{Hexb}^{-/-} \mathrm{FcR} \gamma^{-1-}$ mice than in $\mathrm{Hexb}^{-/-} \mathrm{FcR} \mathrm{\gamma}^{+/+}$ mice. $n=5-10 .{ }^{*} P<0.05$, Student's $t$ test. 
a

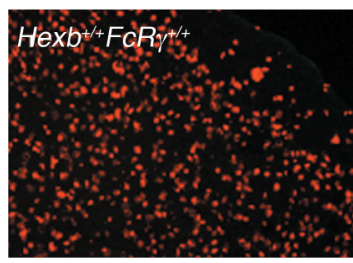

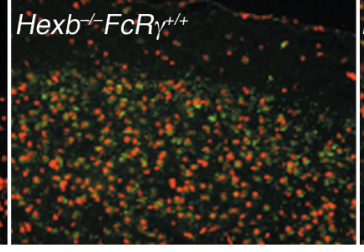

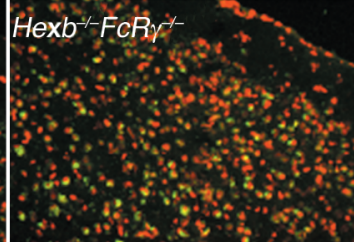

b

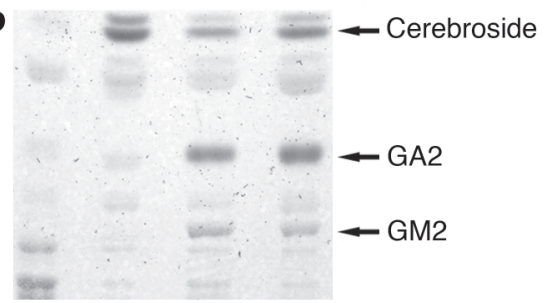

Figure 4

Analysis of ganglioside storage. (a) Immunohistochemical detection of GM2 in the cerebral cortex of a 14-week-old mouse. Both $H e x b^{-/-} F c R \gamma^{\prime /}$ and Hexb $b^{-/-} F c R \gamma^{-1}$ mice, but not WT $\left(H e x b^{+/+} F c R \gamma^{t /+}\right)$, showed GM2 storage in the cerebellum (stained green or yellow). PI was used for nuclear staining. (b) Extraction and analysis of brain gangliosides. Gangliosides were extracted from the brains of 14-week-old mice and loaded onto HPTLC

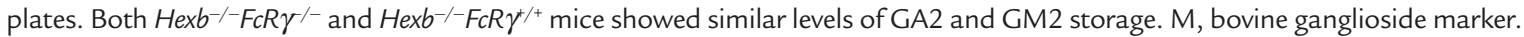

although there was a slight, but statistically significant, elevation in these levels when compared to WT $\left(\mathrm{Hexb}^{+/+} \mathrm{FcR} \gamma^{1 /+}\right)$ mice (Figure 3c).

Patterns and levels of ganglioside accumulation in $\mathrm{Hexb} b^{-1-F c R} \gamma^{-1}$ mice are similar to those seen in $\mathrm{Hexb}^{-1-} \mathrm{FcR} \gamma^{1 /+}$ mice. The levels of accumulated GM2 and GA2 at 14 weeks of age were examined in the brains of $H e x b^{-/-F c R} \gamma^{-1}$ mice. Immunohistostaining of the cerebral cortex with antibodies against GM2 is shown in Figure 4a. $H e x b^{-1-} F c R \gamma^{-1}$ mice revealed no differences in GM2 accumulation compared with $H e x b^{-/-F c R \gamma^{\prime+}}$ mice. We then extracted the gangliosides, and, following analysis by HPTLC, it was revealed that the storage of GM2 and GA2 in the $\mathrm{Hexb}^{-/-} \mathrm{FcR} \gamma^{--}$mice did not change when compared with $\mathrm{Hex} b^{-/-} \mathrm{FcR} \gamma^{1 /+}$ mice (Figure 4b).

Neurological functions are partially restored and life spans are extended in $\mathrm{Hexb} b^{-1-} \mathrm{FcR} \gamma^{--}$mice. Fourteen-week-old $H e x b^{-1-} F c R \gamma^{\prime++}$ mice showed limb flexion when lifted by the tail. On the other hand, both WT and

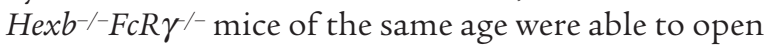
their limbs and move actively (Figure 5a). Clinical scores were judged by (i) the position of the forelimb and hindlimb observed by tail lifting and (ii) the ability to roll over. Both female and male $\mathrm{Hexb}^{-/-} \mathrm{FcR} \gamma^{-1}$ mice always acquired a better score than $H e x b^{-/-F c R \gamma^{\prime+}}$ mice, demonstrating that they had a

\section{Figure 5}

Amelioration of motor defects in $\mathrm{Hexb} b^{-1-} \mathrm{FcR} \gamma^{-1-}$ mice. (a) When suspended by their tails, $H e x b^{-/-} F c R \gamma^{1 /+}$ mice flexed their limbs, whereas $H e x b^{-/-} F c R \gamma^{-1-}$ mice and $H e x b^{+/+} F c R \gamma^{+/+}$mice extended their limbs. (b) Clinical scores were determined by the degree of neurological severity and counted as follows: $0=$ normal, 1 = slight, $2=$ mild, $3=$ moderate, 4 = severe. $\mathrm{Hexb}^{+/+} \mathrm{FcR \gamma ^{+/+ }}, \mathrm{Hexb^{-/- }} \mathrm{FcR \gamma}^{+/+}$, and $\mathrm{Hexb}^{-/-} \mathrm{FcR \gamma ^{-- }}$ mice were tested from 10 to 15 weeks. $n=5-10 .{ }^{*} P<0.05$, Student's

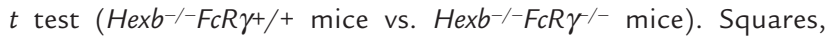
$H e \times b^{-/-} F c R \gamma^{+/+}$mice; diamonds, $H e x b^{+/+} F c R \gamma^{+/+}$mice; triangles, $H e x b^{-1-} F c R \gamma^{-1}$ mice. (c) Rotorod testing was performed by counting the mean time to fall from the rotating rod. $n=5-10$. ${ }^{*} P<0.05$, Stu-

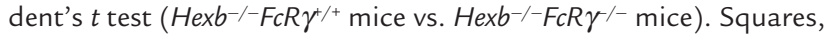
$H e x b^{-/-} F c R \gamma^{+/+}$mice; diamonds, $H e x b^{+/+} F c R \gamma^{+/+}$mice; triangles, Hexb-/-FcR $\gamma^{-1-}$ mice. distinctly milder form of the disease with far less severe neuropathy (Figure 5 b).

A rotorod test, which measures balance and mobility, was performed on $\mathrm{Hexb}^{-/-} \mathrm{FcR} \gamma^{/-}$mice from 10 to 15 weeks of age (Figure 5c). Both female and male $H e x b^{-1-} F c R \gamma^{--}$mice constantly attained better performances than $H e x b^{-/-F c R \gamma^{1 /+}}$ mice during this test.

\section{a}
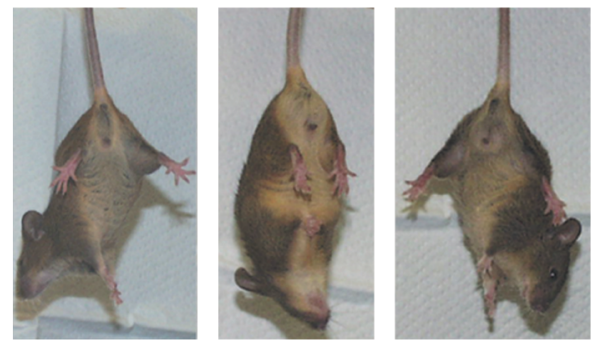

b $\mathrm{Hexb}^{+++} \mathrm{FcR \gamma} \gamma^{+/+}$

$\mathrm{Hexb}^{-/-} \mathrm{FcR} \gamma^{+/+} \mathrm{Hexb}^{-/-} \mathrm{FcR} \gamma^{--}$
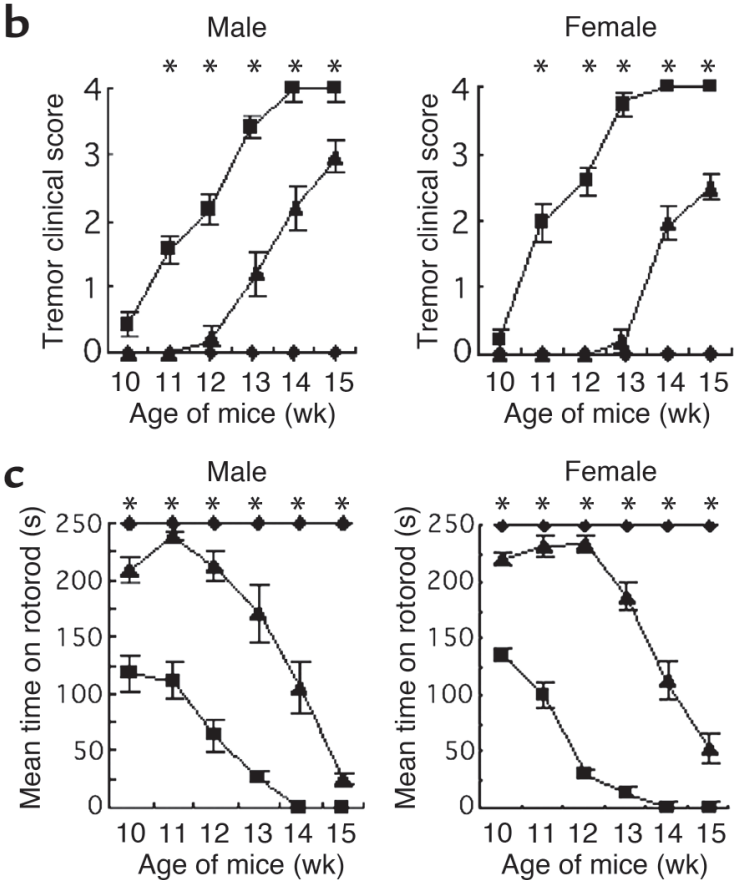

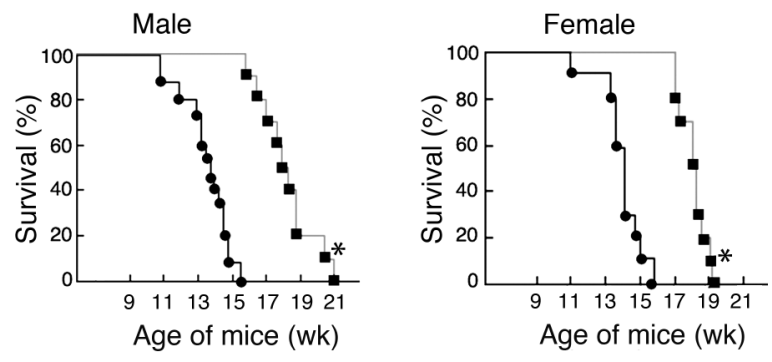

Figure 6

Survival of Hexb $b^{-1-} \mathrm{FcR} \gamma^{-1-}$ mice. Male and female $\mathrm{Hexb}^{-1-} \mathrm{FcR \gamma ^{-1 }}$ mice (squares, $n=10$ ) and $H e x b^{-/-F c R \gamma^{+/+}}$mice (circles, $n=10-15$ ) were monitored daily. ${ }^{*} P<0.0005$, Student's $t$ test.

The performance of the Hexb $b^{-1-} F c R \gamma^{/-}$mice was consistent up to the 12-week point but deteriorated gradually thereafter. The mean survival time of both male and female $H e x b^{-/-} F c R \gamma^{\dagger++}$ mice was 102 days (86-115 days, respectively), whereas that of male and female $H e x b^{-1-} F c R \gamma^{\prime-}$ mice was 130 days (116-145 days) and 128 days (122-135 days), respectively (Figure 6).

Neurodegeneration of Purkinje cells and apoptosis are less extensive in $\mathrm{Hexb}^{-/-F c R} \gamma^{-/}$mice. Neurodegeneration in $H e x b^{-/-F c R \gamma^{\prime++}}$ and $\mathrm{Hexb}^{-/-} \mathrm{FcR} \gamma^{--}$mice was examined in the cerebella of 14-week-old animals. As shown in Figure 7, Purkinje cells of $\mathrm{Hexb}^{-/-} F \mathrm{R} R \gamma^{/ /+}$mice of the same age were either lost or had degenerated. In contrast,

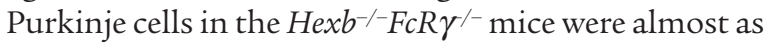
well preserved as in WT $\left(H e x b^{+/+} F c R \gamma^{1 /+}\right)$ mice. We also assessed the nuclei of the Purkinje cells by PI staining. In $\mathrm{Hexb}^{-/-} \mathrm{FcR} \gamma^{/ /}$mice, the nuclei appeared smaller and degenerated, whereas in $H e x b^{-/-} F c R \gamma^{\prime-}$ mice, the nuclei appeared morphologically similar to WT. Based upon these findings, the degeneration of Purkinje cells in

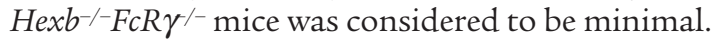

We next evaluated apoptosis in the brains of 14-weekold mice (Figure 8). Using TUNEL assays, positive cells suggestive of apoptosis were found in the thalamus of $H e x b^{-/-} F c R \gamma^{\prime /+}$ mice (Figure 8a), whereas in $H e x b^{-/-F c R} \gamma^{/-}$ and WT mice, these TUNEL-positive cells were barely detectable. The number of TUNEL-positive cells in a sagittal section was counted (Figure $8 \mathrm{~b}$ ) and was shown to have decreased significantly in $\mathrm{Hexb}^{-/}-\mathrm{FcR} \gamma^{-}$- mice when compared with $H e x b^{-1-} F c R \gamma^{\prime+}$ mice. Apoptotic changes were also evaluated by a DNA ladder test, in which extracted DNA from mouse brain was run on an agarose gel. DNA from $H e x b^{-/} F c R \gamma^{1 /+}$ mouse brain appeared as a ladder with a smear pattern, whereas in $H e x b^{-1-} F c R \gamma^{\prime-}$ mice, a very faint ladder/smear pattern was visible. From these findings, we conclude that apoptosis did not occur extensively in the brain of the $\mathrm{Hex}^{-/-F c R \gamma^{-}}$ mice when compared with $H e x b^{-1-F c R \gamma^{\prime /}}$ mice.

IgG deposition were also observed in the brain of an $S D$ patient. To determine whether autoimmune reactions were also present in human SD, we obtained autopsied tissue samples from the brain of an SD patient and examined them histologically. Neurons in the cerebellum were found to be distended with evidence of ganglioside storage (Figure 9, a and b). IgG deposition was investigated in the cerebellum of this patient and was indeed evident in neurons, similar to the findings observed in $\mathrm{Hexb}^{-/-}$mice (Figure 9, $\mathrm{c}$ and d). Thus, the same immunological mechanisms found in the mouse may also be involved in human SD.

\section{Discussion}

LSDs comprise a family of more than 40 different disorders that result from deficiencies in various lysosomal enzymes (1). These disorders have much in common, such as pathological lysosomal swelling due to lysosomal storage of undegraded substrates and a mannose-6-phosphate receptor-mediated enzyme recapture system that has been targeted in the development of gene therapy for these diseases (20-23). Although emerging genetic technologies have uncovered the primary genetic defects of many LSDs, many uncertainties remain concerning the mechanism(s) leading to cell injury and disease progression. Extreme lysosomal swelling may lead to malfunction of cells that would delay signal transduction, but it is questionable whether this alone can account for the severe phenotypes seen in patients. In this study we investigated the pathogenesis of SD mice from the perspective of autoimmune features.

In the SD mouse model, both GM2 and GA2 accumulate in lysosomes due to defects in Hex's A and B (4). These gangliosides are abundant in the neurons

\footnotetext{
Figure 7

Reduction in the neurodegeneration of Purkinje cells in $\mathrm{Hexb}^{-/-} \mathrm{FcR} \gamma^{-1-}$ mice and 14-week-old Hexb $b^{-/-} \mathrm{F} c R \gamma^{1 /+}$ mice, but not $\mathrm{Hexb}^{-/-} \mathrm{FcR \gamma ^{-/ }}$ or $H e x b^{+/+} F c R \gamma^{+/+}$, displayed neurodegeneration and shrinkage of nuclei in Purkinje cells. Arrows indicate nuclei of Purkinje cells. H\&E stain, original magnification, $\times 100$. PI stain, original magnification, $\times 600$. ML, molecular layer, Pc, Purkinje cells, GL, granular layer.
}
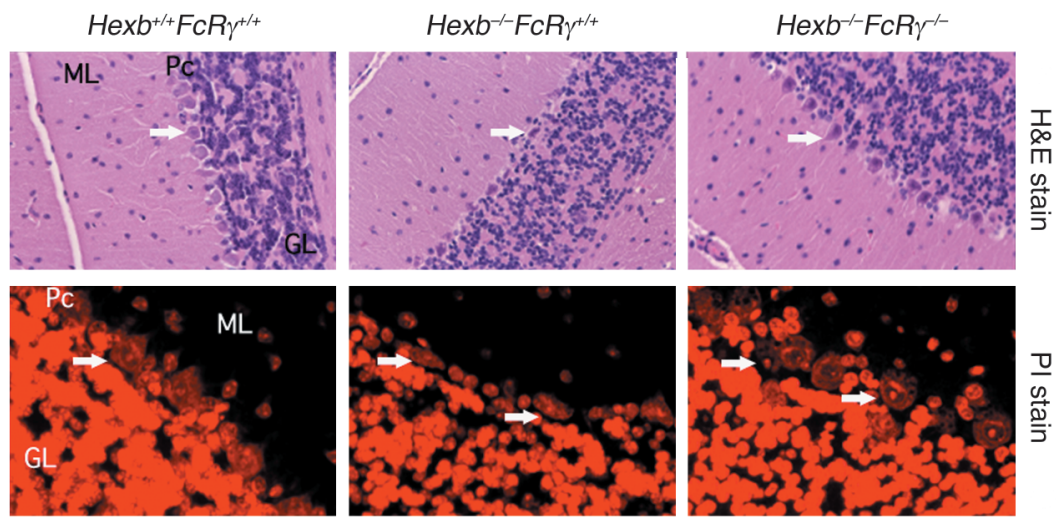


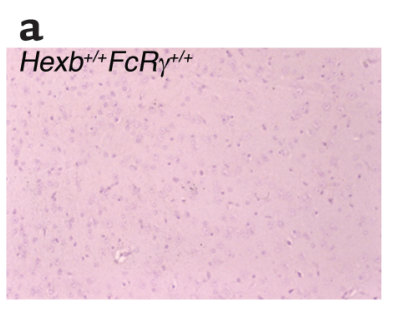

b

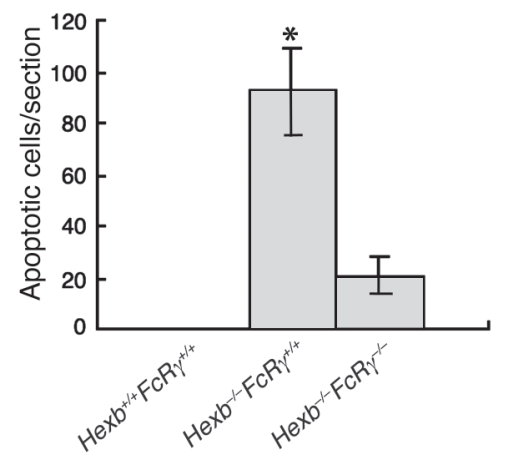

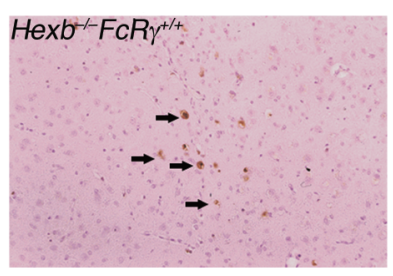

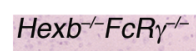

c

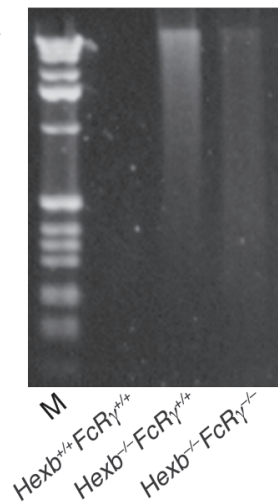

\section{Figure 8}

Reduction of apoptosis in the brain of $\mathrm{Hexb}^{-/-} \mathrm{FCR}_{\mathrm{C}}{ }^{-1-}$ mice. (a) At 14 weeks, Hexb ${ }^{-1-} F c R \gamma^{\prime-}$ mice were analyzed by in situ TUNEL analysis, and compared with $\mathrm{Hexb}^{-/-} \mathrm{FcR} \gamma^{+/+}$and $\mathrm{Hexb^{+/+ }} F c R \gamma^{1 /+}$ mice. Arrows indicate apoptotic cells. Original magnification, $\times 100$ (thalamus). (b) Numbers of apoptotic (TUNEL positive) cells were counted in a sagittal section of the brain. Data are mean $\pm \operatorname{SEM}(n=4)$. ${ }^{*} P<0.001$, Student's $t$ test. (c) DNA laddering in the brain from $\mathrm{Hexb}^{+/+} \mathrm{FcR} \mathrm{\gamma}^{+/+}, \mathrm{Hexb}^{-/-} \mathrm{FcR \gamma ^{-/ }}$, and $H e x b^{-/-} F c R \gamma^{\dagger /+}$ mice. One microgram of brain DNA was subjected to electrophoresis on a $2 \%$ agarose gel and stained with ethidium bromide. The lane at the left of the panel shows a 1-kb DNA ladder. of the CNS but are also present to some degree in visceral organs such as the liver, spleen, and thymus. In our study, we hypothesized that accumulated gangliosides might not be easily degraded and cleared due to the absence of Hex's. Thus, such undegraded substrates could potentially be targeted by the immune system, resulting in an autoimmune response. Similar mechanisms have been suggested in some cases of systemic lupus erythematosus (SLE), where low-serum DNase I activity, due to DNase I mutations, may result in undegraded nuclear DNA and hence, antinuclear DNA antibody formation (24). Indeed, DNase I-knockout mice do develop SLE-like glomerulonephritis with the formation of anti-chromatin autoimmunity (25). The antigenicity of gangliosides is further indicated by GuillainBarré syndrome, amyotrophic lateral sclerosis, and Fisher syndrome (11-13), and we therefore postulated that accumulated gangliosides in SD mice might serve as autoantigens.

An initial series of experiments demonstrated increased levels of serum anti-GM2 and anti-GA2 antibodies in 14-week-old SD mice that showed advanced illness. Immunohistochemical studies of these mice also revealed IgG deposition in neurons of the CNS. IgG deposition in CNS neurons may be consistent with a recent report showing increased $\mathrm{BBB}$ permeability not only in SD mice but also in GM1 gangliosidosis mice (10). Although it is still uncertain whether antiganglioside antibodies accumulate in neurons, serum from SD mice successfully transferred IgG deposition to the CNS neurons of SCID mice that had undergone D-mannitol treatment to disrupt the BBB. These data strongly suggested the presence of specific autoimmunity in SD mice. We hypothesized that antigen-antibody complexes on cell surfaces would trigger type II hyper- sensitivity reactions with microglial activation via FcR $\gamma$ because microglial activation has been reported in SD mice $(8,9)$, and $F c R \gamma$-knockout mice are resistant to the induction or spontaneous onset of various autoimmune diseases (26-28).

To further clarify this hypothesis, we generated and

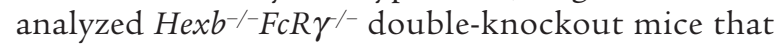
lack the FcR $\gamma$ in SD mice. In the Hexb $b^{-1-} F c R \gamma^{\prime-}$ mice, amelioration of neurological symptoms, life span increases, reduction in the degeneration of Purkinje cells, decreased levels of neuronal cell death, and decreased levels of anti-GA2 antibodies were all observed. However, as found in BMT experiments, the accumulation of gangliosides in SD mice was also evi-
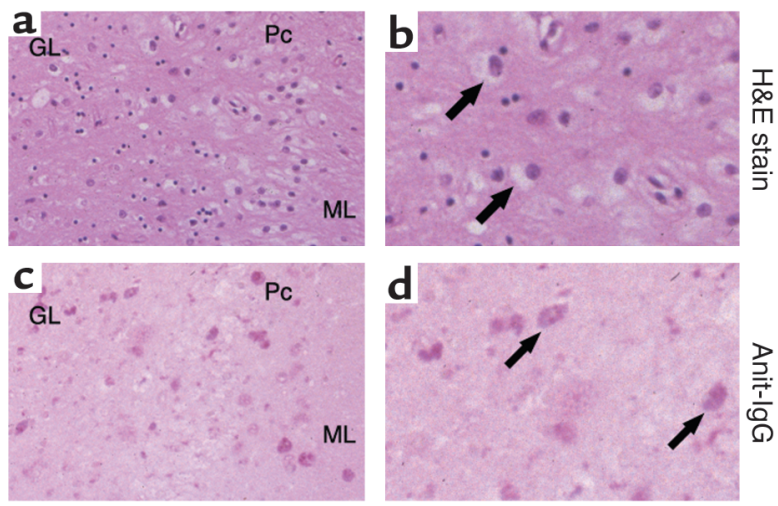

\section{Figure 9}

IgG deposition in the cerebellar neurons of an SD patient. ( $\mathbf{a}$ and $\mathbf{b}$ ) H\&E staining of cerebellar sections showed cells with typical ballooning. Arrows indicate enlarged cells with ganglioside storage. (c and d) Immunohistochemical staining of the cerebellum with alkaline phosphatase-labeled anti-lgG antibody. Arrows indicate cells with $\lg G$ deposition (stained as red). (a and c) Original magnification, $\times 200$; (b and $\mathbf{d}$ ) Original magnification, $\times 400$. ML, molecular layer; Pc, Purkinje cells; GL, granular layer. 
dent in $\mathrm{Hexb}^{-/-} \mathrm{FcR} \gamma^{/-}$mice (7). These data suggest that this accumulation is one of the factors in the pathophysiology of LSDs.

Recent substantial studies have suggested that inflammatory processes are involved in the development of the neuropathology of SD mice $(8,10)$. Using cDNA microarray analysis, Wada et al. revealed the upregulation of genes related to an inflammatory process dominated by activated microglia and confirmed that there were increased numbers of activated microglia and increased level of TNF- $\alpha$ mRNA in both SD mice and an autopsied human SD patient (8). Gene expression profiling by serial analysis of gene expression in human GM2 gangliosidoses, including Tay-Sachs disease, supported the role of microglial and astroglial activation, and demonstrated the increased expression of death-associated protein 6 (DAXX), which encodes a protein that associates with the Fas receptor and is involved in apoptosis via Fas (9). Moreover, microglial activation accompanied by the production of many other cytokines was previously reported in both SD mice and GM1 gangliosidosis mice (10).

Based on our findings and the reports of other laboratories, we speculate that antibody-ganglioside complexes are important for the induction of microglial activation that is mediated via FcR $\gamma$ in SD mice. Data showing that BMT ameliorates neurologic manifestations in SD mice can be explained by decreased anti-GM2 and anti-GA2 antibody formation, which we have observed (unpublished data). Activated microglia would produce cytokines including TNF- $\alpha$, which is known to induce apoptosis through DAXX $(29,30)$. The neurodegeneration that is observed in Purkinje cells of SD mice may therefore be the result of proinflammatory cytokines from activated microglia, although the precise mechanisms underlying this remain to be determined. Another possible mechanism by which activated microglia may cause SD is through phagocytosis via the FcR $\gamma$ chain. Antibody-ganglioside complexes may become opsonized and could potentially be either phagocytosed or injured by microglia. Apoptotic neurons bearing these antibody-ganglioside immune complexes would therefore be phagocytosed and internalized by microglia. As a result of these mechanisms, neuronal injury, degeneration, and cell death are induced. As a result, microglial activation would accelerate antigen presentation and inflammatory responses that augment increasing levels of autoantibody production. A pathological study is currently ongoing in our laboratory to precisely evaluate microglial activation in $\mathrm{Hexb}^{-/-} \mathrm{FcR} \gamma^{--}$mice. Preliminary results indicate that there is reduced microglial activation, which supports our hypothesis.

Importantly, the autoantibody mechanisms evident in mouse models appear to exist also in human SD. Although we have so far only demonstrated the presence of IgG deposition in a CNS specimen from a sin- gle SD patient, it is highly possible that similar mechanisms exist in other cases of LSDs, including gangliosidoses. The manifestation of these may well depend on both the antigenicity and the levels of antigens. Immunological disturbances of the sphingolipidoses have been reported in some cases of Gaucher disease in which patients have significantly higher concentrations of polyclonal immunoglobulin (31). Gaucher disease mice generated by Mizukami et al. also showed systemic inflammatory changes (32). It would be very interesting in the future to investigate the autoantibody production associated with other LSDs.

Our results clearly demonstrate the involvement of the $F c R \gamma$ gene and the possible role of an autoimmune response in the development of SD. Although much still remains to be elucidated, our present investigation has given us novel insights and furthered our understanding of the pathogenesis of SD. The contribution of autoimmunity to the pathogenesis of LSDs will be an important factor when developing effective new treatments to be used in combination with other therapies such as $\operatorname{BMT}(7,33,34)$, enzyme replacement therapy (35), and substrate deprivation $(33,36)$.

\section{Acknowledgments}

We thank Richard L. Proia for providing the SD mice and Masae Tatematsu for the generous donation of the human SD brain autopsy tissue. We also thank Kyoko Suzuki, Eizo Iseki, Haruto Hojo, Yoji Nagashima, Yoshihiro Kiuchi, Dennis M. Klinman, Rieko Ijiri, Michiko Ehara, and Yoshitsugu Kojima for technical assistance and helpful discussions. This project was carried out with financial support from a grant-in-aid for scientific research from the Japanese Society for the Promotion of Science and a grant in support of Promotion of Research from Yokohama City University.

\footnotetext{
1. Neufeld, E.F. 1991. Lysosomal storage diseases. Annu. Rev. Biochem. 60:257-280.

2. Gravel, R.A., et al. 2001. The GM2 gangliosidoses. In The metabolic and molecular basis of inherited disease. C.R. Scriver et al., editors. McGraw-Hill. New York, New York, USA. 3827-3876.

3. Yamanaka, S., Johnson, O.N., Norflus, F., Boles, D.J., and Proia, R.L. 1994. Structure and expression of the mouse beta-hexosaminidase genes, Hexa and Hexb. Genomics. 21:588-596.

4. Sango, K., et al. 1995. Mouse models of Tay-Sachs and Sandhoff diseases differ in neurologic phenotype and ganglioside metabolism. Nat. Genet. 11:170-176.

5. Phaneuf, D., et al. 1996. Dramatically different phenotypes in mouse models of human Tay-Sachs and Sandhoff diseases. Hum. Mol. Genet. 5:1-14

6. Huang, J.Q., et al. 1997. Apoptotic cell death in mouse models of GM2 gangliosidosis and observations on human Tay-Sachs and Sandhoff diseases. Hum. Mol. Genet. 6:1879-1885.

7. Norflus, F., et al. 1998. Bone marrow transplantation prolongs life span and ameliorates neurologic manifestations in Sandhoff disease mice. J. Clin. Invest. 101:1881-1888.

8. Wada, R., Tifft, C.J., and Proia, R.L. 2000. Microglial activation precedes acute neurodegeneration in Sandhoff disease and is suppressed by bone marrow transplantation. Proc. Natl. Acad. Sci. U. S. A. 97:10954-10959.

9. Myerowitz, R., et al. 2002. Molecular pathophysiology in Tay-Sachs and Sandhoff diseases as revealed by gene expression profiling. Hum. Mol. Genet. 11:1343-1350.
} 
10. Jeyakumar, M., et al. 2003. Central nervous system inflammation is a hallmark of pathogenesis in mouse models of GM1 and GM2 gangliosidosis. Brain. 126:974-987.

11. Yuki, N. 1999. Pathogenesis of Guillain-Barre and Miller Fisher syndromes subsequent to Campylobacter jejuni enteritis. Jpn. J. Infect. Dis. 52:99-105.

12. Niebroj-Dobosz, I., Jamrozik, Z., Janik, P., Hausmanowa-Petrusewicz, I., and Kwiecinski, H. 1999. Anti-neural antibodies in serum and cerebrospinal fluid of amyotrophic lateral sclerosis (ALS) patients. Acta Neurol. Scand. 100:238-243.

13. O'Hanlon, G.M., et al. 2000. Peripheral neuropathy associated with antiGM2 ganglioside antibodies: clinical and immunopathological studies. Autoimmunity. 32:133-144.

14. Takai, T., Li, M., Sylvestre, D., Clynes, R., and Ravetch, J.V. 1994. FcR gamma chain deletion results in pleiotrophic effector cell defects. Cell. 11:519-529.

15. Sango, K., Yamanaka, S., Ajiki, K., Tokashiki, A., and Watanabe, K. 2002 Lysosomal storage results in impaired survival but normal neurite outgrowth in dorsal root ganglion neurons from a mouse model of Sandhoff disease. Neuro. Pathol. Appl. Neurobiol. 28:1-17.

16. Tatematsu, M., et al. 1981. Sandhoff disease. Acta Pathol. Jpn. 31:503-512.

17. Taki, T., Nishiwaki, S., Ishii, K., and Handa, S. 1990. A simple and specific assay of glycosyltransferase and glycosidase activities by an enzymelinked immunosorbent assay method, and its application to assay of galactosyltransferase activity in sera from patients with cancer. J. Biochem. (Tokyo). 107:493-498.

18. Bhattacharjee, A.K., Nagashima, T., Kondoh, T., and Tamaki, N. 2001. The effects of the $\mathrm{Na}(+) / \mathrm{Ca}(++)$ exchange blocker on osmotic bloodbrain barrier disruption. Brain Res. 11:157-162.

19. Rapoport, S.I., Fredericks, W.R., Ohno, K., and Pettigrew, K.D. 1980. Quantitative aspects of reversible osmotic opening of the blood-brain barrier. Am. J. Physiol. 238:R421-R431.

20. Yamaguchi, A., et al. 2003. Plasmid-based gene transfer ameliorates visceral storage in a mouse model of Sandhoff disease. J. Mol. Med. 81:185-193.

21. Neufeld, E.F., and Fratantoni, J.C. 1970. Inborn errors of mucopolysaccharide metabolism. Science. 169:141-146.

22. Yew, N.S., and Cheng, S.H. 2001. Gene therapy for lysosomal storage disorders. Curr. Opin. Mol. Ther. 3:399-406.

23. Bou-Gharios, G., Abraham, D., and Olsen, I. 1993. Lysosomal storage diseases: mechanisms of enzyme replacement therapy. Histochem. $J$. 25:593-605.

24. Walport, M.J. 2000. Lupus, DNase and defective disposal of cellular debris. Nat. Genet. 25:135-136.

25. Napirei, M., et al. 2000. Features of systemic lupus erythematosus in Dnase1-deficient mice. Nat. Genet. 25:177-181.

26. Takai, T. 2002. Roles of Fc receptors in autoimmunity. Nat. Rev. Immunol. 2:580-592.

27. Ravetch,J.V., and Bolland, S. 2001. IgG Fc receptors. Annu. Rev. Immunol. 19:275-290.

28. Abdul-Majid, K.B., et al. 2002. Fc receptors are critical for autoimmune inflammatory damage to the central nervous system in experimental autoimmune encephalomyelitis. Scand. J. Immunol. 55:70-81.

29. Shinoda, S., et al. 2003. Formation of a tumour necrosis factor receptor 1 molecular scaffolding complex and activation of apoptosis signal-regulating kinase 1 during seizure-induced neuronal death. Eur. J. Neurosici. 17:2065-2076.

30. Yang, X., Khosravi-Far, R., Chang, H.Y., and Baltimore, D. 1997. Daxx, a novel Fas-binding protein that activates JNK and apoptosis. Cell. 27:1067-1076.

31. Marti, G.E., et al. 1988. Polyclonal B-cell lymphocytosis and hypergammaglobulinemia in patients with Gaucher disease. Am. J. Hematol. 29:189-194.

32. Mizukami, H., et al. 2002. Systemic inflammation in glucocerebrosidasedeficient mice with minimal glucosylceramide storage. J. Clin. Invest. 109:1215-1221. doi:10.1172/JCI200214530.

33. Jeyakumar, M., et al. 2001. Enhanced survival in Sandhoff disease mice receiving a combination of substrate deprivation therapy and bone marrow transplantation. Blood. 97:327-329.

34. Krivit, W., Peters, C., and Shapiro, E.G. 1999. Bone marrow transplantation as effective treatment of central nervous system disease in globoid cell leukodystrophy, metachromatic leukodystrophy, adrenoleukodystrophy, mannosidosis, fucosidosis, aspartylglucosaminuria, Hurler, Maroteaux-Lamy, and Sly syndromes, and Gaucher disease type III. Curr Opin. Neurol. 12:167-176.

35. Barton, N.W., et al. 1991. Replacement therapy for inherited enzyme deficiency-macrophage-targeted glucocerebrosidase for Gaucher's disease. N. Engl. J. Med. 23:1464-1470.

36. Tifft, C.J., and Proia, R.L. 2000. Stemming the tide: glycosphingolipid synthesis inhibitors as therapy for storage diseases. Glycobiology. 10:1249-1258. 